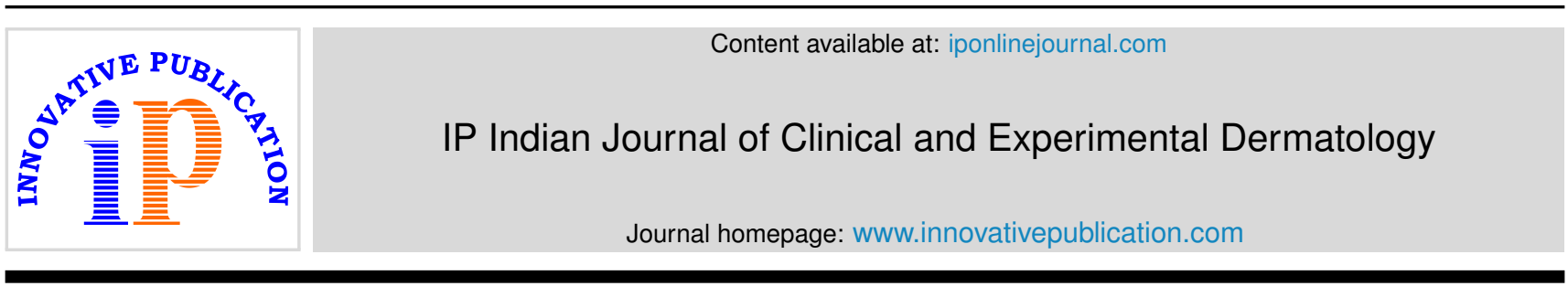

Original Research Article

\title{
Comparison of the diagnostic efficacy of Tzanck smear with histopathology and direct immunofluorescence in immunobullous disorders
}

\author{
S Aneesh ${ }^{1}$, BN Raghavendra ${ }^{1, *}$, Krishnan Aaryambika ${ }^{1}$, Anjan Kumar Patra ${ }^{1}$, \\ KD Bijina $^{1}$, Sandhaya ${ }^{1}$ \\ ${ }^{1}$ Dermatology, MVJ Medical College and Research Hospital, Hoskote, Karnataka, India
}

\section{A R T I CLE I N F O}

Article history:

Received 26-07-2019

Accepted 06-08-2019

Available online 14-09-2019

Keywords:

Direct immunofluorescence

Histopathology

Immunobullous disorders

Tzanck smear.

\begin{abstract}
A B S T R A C T
Introduction: The immunobullous disorders have severe impact on the patients and their family and have severe economic and health related consequences associated with pain, agony and loss of manpower. Tzanck smear being a bed side test can aid in establishing the clinical diagnosis with ease and rapidity and can serve as an adjunct to routine histological study. This study was undertaken to assess the utility of Tzanck test for early diagnosis of various immunobullous disorders and correlate the findings with histopathology and direct immunofluorescence.

Objectives: 1.To study Tzanck smear, histopathology, and immunofluorescence patterns in immunobullous disorders. 2.To evaluate diagnostic efficacy and sensitivity of Tzanck smear findings in comparison to histopathology and direct immunofluorescence in clinically suspected cases of immunobullous disorders. Materials and Methods: A total of 38 cases clinically diagnosed immunobullous disorders were appraised during the study. The patients were subjected to Tzanck smear, skin biopsy for histopathology and direct immunofluorescence. The specimen was sent in $10 \%$ buffered formalin and normal saline for routine histological analysis and DIF respectively to the department of Pathology. The resulting data was tabulated and statistically analysed for comparison using sensitivity test.

Conclusion: Tzanck smear served as a simple, rapid and non invasive bedside diagnostic tool, which was cost effective and yield ed quick results. It can be concluded that in pemphigus group of disorders, Tzanck smear is $100 \%$ sensitive, can yield quick results and can serve as first line of investigation rather than histopathology and DIF which is expensive and takes time for reporting.

Results: Pemphigus vulgaris was the most common immunobullous disease seen in this study, followed by bullous pemphigoid. Tzanck smear served as a rapid diagnostic tool and showed $100 \%$ sensitivity in all the pemphigus group of disorders which was compared with histopathological findings, which also showed $100 \%$ sensitivity whereas DIF was positive in $93.54 \%$ of cases.
\end{abstract}

(C) 2019 Published by Innovative Publication.

\section{Introduction}

The immunobullous disorders are a group of autoimmune diseases in which components of the epidermis and basement membrane zone are the focus of attack, resulting in the formation of cutaneous and mucosal blisters. The target antigens are components of desmosomes or the functional unit of the basement membrane zone known as the adhesion complex. ${ }^{1}$ Early diagnosis and treatment of

\footnotetext{
* Corresponding author.

E-mail address: raghavderm@gmail.com (B. N. Raghavendra).
}

these severe and potentially life-threatening disorders is essential in preventing the consequences. Tzanck smear is a simple, economical and rapid bed side technique which can be used in early diagnosis of immunobullous disorders, whereas histopathology and direct immunofluorescence, though specific, are time consuming and expensive. ${ }^{2}$ This study was undertaken to assess the utility of Tzanck test in early diagnosis of various immunobullous disorders and correlate the findings with histopathology and direct immunofluorescence. 


\section{Material and Method}

In this prospective, observational, hospital based study conducted in a tertiary care hospital, Thirty eight patients presenting with immunobullous disorders attending the outpatient department of dermatology were enrolled after obtaining a written consent. The study was conducted between September 2016 and June 2018 after approval from institutional ethical committee. The objective was to study Tzanck smear, histopathology, and immunofluorescence patterns in immunobullous disorders and to evaluate the diagnostic efficacy and sensitivity of Tzanck smear findings in comparison to histopathology and direct immunofluorescence in clinically suspected cases of immunobullous disorders.

A detailed clinical history was taken including age, sex, occupation, site, and duration of symptoms. The case

$\mathrm{s}$ were subjected to Tzanck smear, biopsy for histopathology and direct immunofluorescence. Tzanck smear was taken from all cases, stained with Giemsa stain for cytological examination. Two skin biopsies were taken from each case and specimen was sent to laboratory in $10 \%$ formalin and phosphate buffer saline for routine histopathological examination and DIF respectively. The resulting data was tabulated and statistically analysed for comparison using sensitivity test.

\section{Results}

A total of 38 cases of immunobullous disorders, with 17 females $(44.73 \%)$ and 21 males $(55.26 \%)$ were included in the studyTable 1 . The age ranged between 17 and 90 years with mean value of 44.47 years. In this study, the predominant number of cases were in the age group between $30-60$ yearsTable 2 . In the present study, majority of the cases were $\mathrm{p}$ emphigus vulgaris 31 cases $(81.57 \%)$ followed by bullous pemphigoid (13.15\%), and dermatitis herpetiformis 2 cases $(5.26 \%)$ Table 3.

In pemphigus vulgaris group, Nikolsky sign was positive in $77.41 \%$ and Bulla spread sign was positive in $51.61 \%$ of the cases. Tzanck smear was positive for acantholytic cells in all the cases of pemphigus vulgaris $(100 \%)$ with lymphocytes being the predominant infiltrate present in $77.41 \%$ Figure 1. In histopathology, suprabasal split was seen in all cases $(100 \%)$ Figure 2 and acantholytic cells were seen $90.32 \%$ of the cases. IgG and C3 deposits were seen on DIF in $93.54 \%$ of cases Table 4.

In all the cases of bullous pemphigoid, acantholytic cells were absent and eosinophils were found to be the predominant infiltrates in Tzanck smear. In histopathology, subepidermal splitFigure 3 and DIF showed linear deposits of $\mathrm{IgG}$ and $\mathrm{C} 3$ in all the cases. Acantholytic cells were conspicuously absent in case of dermatitis herpetiformis with presence of predominant neutrophilic infiltration in Tzanck smear. A subepidermal split was observed in all cases and DIF showed linear IgA and C3 depositsTable 6Figure 4.

Table 1: Sex incidence

\begin{tabular}{lll}
\hline Sex & Number of cases & Percentage \\
Male & 21 & 55.2 \\
Female & 17 & 44.73 \\
Total & 38 & 100 \\
\hline
\end{tabular}

Table 2: Age distribution

\begin{tabular}{lll}
\hline Age in years & Number of cases & Percentage \\
$<30$ & 5 & 13.15 \\
$30-60$ & 25 & 65.78 \\
$>60$ & 8 & 21.05 \\
Total & 38 & 100 \\
\hline
\end{tabular}

Table 3: Clinical Diagnosis

\begin{tabular}{lll}
\hline Clinical Diagnosis & Number of cases & Percentage \\
Pemphigus vulgaris & 31 & 81.57 \\
Bullous pemphigoid & 5 & 13.15 \\
Dermatitis & 2 & 5.26 \\
herpetiformis & & \\
Total & 38 & 100 \\
\hline
\end{tabular}

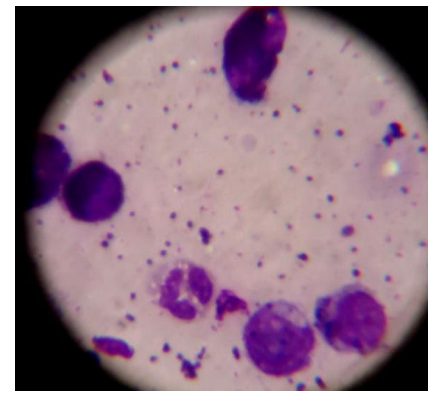

Fig. 1: Tzanck smear showing acantholytic cells, and lymphocytes

\section{Discussion}

The maximum number of patients in this study were in the age group of 30 to 60 years $(65.78 \%)$. Out of 31 cases of pemphigus vulgaris, maximum number of patients were in age group of 30 to 60 years $(77.41 \%)$, where as in the study done by Handa et $\mathrm{al}^{3}, 57.6 \%$ of pemphigus vulgaris patients were in the age group of 11 to 15 years.

In this study, $44.73 \%$ of the patients were females and $55.26 \%$ were males with a male to female ratio of 1.23 : 1 which was in accordance with the study by Leena et al and Arya et al. ${ }^{4,5}$ Out of 31 cases of pemphigus vulgaris, $17(54.83 \%)$ were male, $14(45.16 \%)$ were female with male to female ratio of 1.21:1. This was in accordance with 
Table 4: Comparison of Tzanck smear, histopathology and DIF in pemphigus vulgaris

\begin{tabular}{llll}
\hline \multicolumn{1}{c}{ Pemphigus vulgaris } & Number of cases with positive findings & Total cases & Percentage \\
$\begin{array}{l}\text { Tzanck smear (Acantholytic } \\
\text { cells) }\end{array}$ & 31 & 31 & 100 \\
$\begin{array}{l}\text { Histopathology (Acantholytic } \\
\text { cells) }\end{array}$ & 31 & 31 & 100 \\
$\begin{array}{l}\text { Histopathology (Suprabasal } \\
\text { split) }\end{array}$ & 28 & 31 & 90.32 \\
DIF (IgG and C3) & 29 & 31 & 93.54 \\
\hline
\end{tabular}

Table 5: Comparison of Tzanck smear, histopathology and DIF in Bullous pemphigoid

\begin{tabular}{llll}
\hline Pemphigus vulgaris & $\begin{array}{l}\text { Number of cases with positive } \\
\text { findings }\end{array}$ & Total cases & Percentage \\
Histopathology (Acantholytic cells) & 0 & 5 & 0 \\
Histopathology (Subepidermal split) & 5 & 5 & 100 \\
DIF (IgG and C3) & 5 & 5 & 100 \\
\hline
\end{tabular}

Table 6: Comparison ofTzanck smear, Histopathology and DIF in Dermatitis herpetiformis

\begin{tabular}{llll}
\hline $\begin{array}{l}\text { Pemphigus vulgaris } \\
\text { Histopathology (Acantholytic }\end{array}$ & Number of cases with positive findings & Total cases & Percentage \\
$\begin{array}{l}\text { cells) } \\
\begin{array}{l}\text { Histopathology (Subepidermal } \\
\text { split) }\end{array}\end{array}$ & 2 & 2 & 0 \\
DIF (IgA and C3) & 2 & 2 & 100 \\
\hline
\end{tabular}

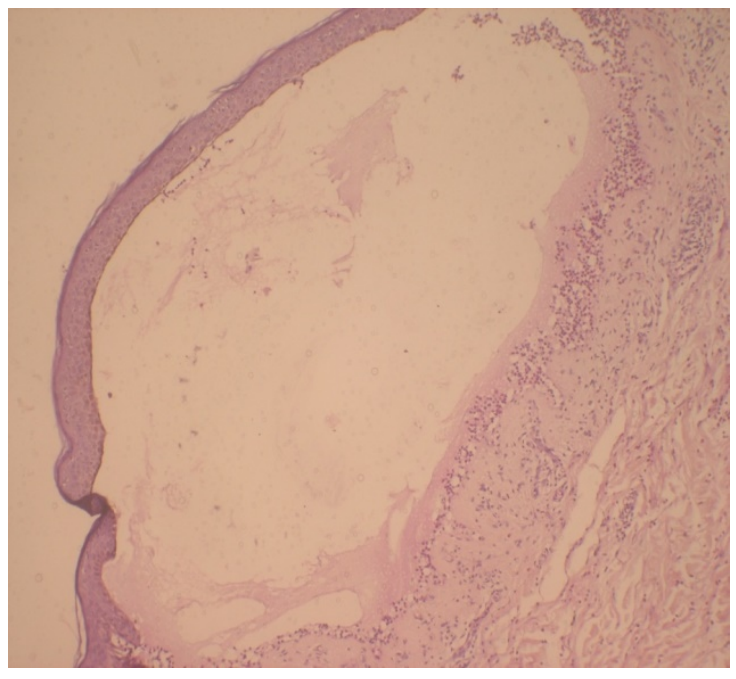

Fig. 2: Photo micrograph of $\mathrm{H} \& \mathrm{E}$ stain, low power view, suprabasal bulla in pemphigus vulgaris

various studies which found the ratios ranging from 1:1.04 to $1: 1.6^{6-8}$

In this study, Tzanck smear showed acantholytic cells in $81.57 \%(31 / 38)$ of all the immunobullous disorders. Acantholytic cells were present in all cases of pemphigus vulgaris $100 \%$ (31/31 cases) which was similar to a study by Leena et $\mathrm{al}^{9}$ and were negative in all the cases of bullous pemphigoid and dermatitis herpetiformis. Deepti et al ${ }^{10}$ in her cohort found neutrophils in $58.8 \%$ cases of pemphigus vulgaris, with absent lymphocytes in pemphigus bullous pemphigoid and dermatitis herpetiformis. A predominant infiltrate of neutrophils was observed in $100 \%$ cases of dermatitis herpetiformis, and eosinophils were found in $76.4 \%$ cases of bullous pemphigoid. The present cohort showed predominant lymphocytic infiltration in $77.41 \%$, cases of pemphigus, $80 \%$ of dermatitis herpetiformis and $50 \%$ cases of bullous pemphigoid. Neutrophilic infiltration was predominantly observed in cases of dermatitis herpetiformis $(100 \%), 41.93 \%$ of pemphigus vulgaris and $80 \%$ of bullous pemphigoid. Eosinophilic infiltration was noted in all the cases of bullous pemphigoid, and in $50 \%$ cases of dermatitis herpetiformis. It was absent in all the cases of pemphigus vulgaris which was in accordance with study by Deepti et al. ${ }^{10}$

In this study, suprabasal split was seen in all cases of pemphigus vulgaris $(100 \%)$ which was comparable with study by Arya et al $(81.4 \%){ }^{5}$ The subepidrmal split was seen in all cases of bullous pemphigoid and dermatitis herpetiformis $(100 \%)$ which was in accordance with studies by Leena et $\operatorname{al}(100 \%)^{4}$ and Banu $\mathrm{L}$ et al(100\%). ${ }^{11}$ In $90.32 \%$ cases of pemphigus vulgaris acantholytic cells were noted, while it was completely absent in cases of bullous pemphigoid and dermatitis herpetiformis. A study by Deepti et al ${ }^{10}$ had similar findings.

The immune complex deposits of $\mathrm{IgG}$ and $\mathrm{C} 3$ were seen in $93.54 \%$ of pemphigus vulgaris and in $100 \%$ cases of bullous pemphigoid. All the cases of dermatitis 


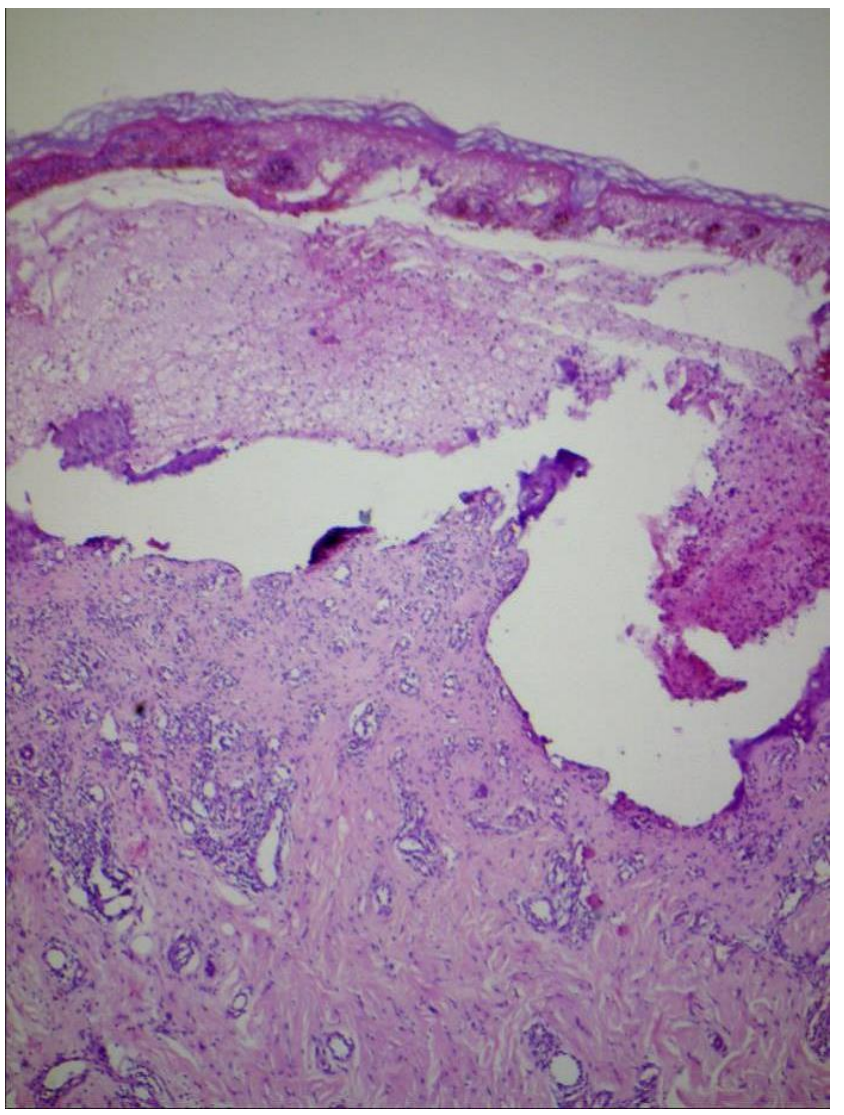

Fig. 3: Photo micrograph of $\mathrm{H} \& \mathrm{E}$ stain, low power view showing subepidermal bulla in bullous pemphigoid

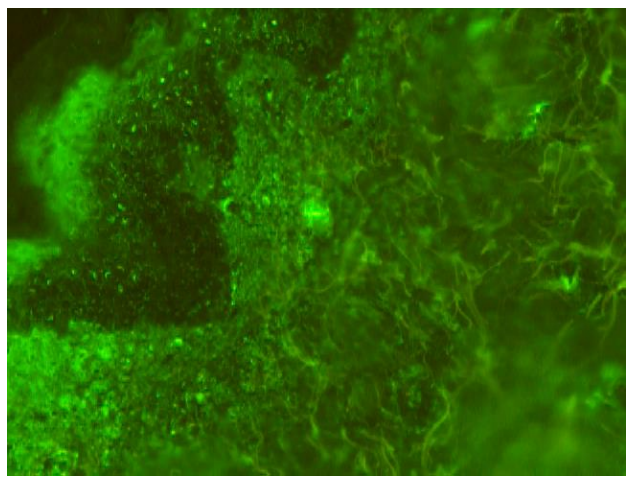

Fig. 4: DIF- showing linear immune complex deposits along dermal papillae herpetiformis showed deposits of $\operatorname{Ig} \mathrm{A}$ and $\mathrm{C} 3$. The above findings were comparable with various studies. In a study by Deepti et al ${ }^{10}$, DIF was positive in $94.11 \%$ (16/17) cases of pemphigus, in a study by Chams-Davatchi $\mathrm{C}$ et al, ${ }^{12}$ DIF was positive in 93.2\% (389/417) cases of pemphigus vulgaris. Kaur JS et al ${ }^{13}$, noted DIF to be positive in $100 \%$ cases of pemphigus and Inchara $\mathrm{K}$ et al, ${ }^{14}$ reported DIF to be positive in $89.7 \%$ (26/29) cases of pemphigus vulgaris.

The studies by Deepti et al ${ }^{10}$ and Deepthi PK et al ${ }^{15}$ reported DIF to be positive in $100 \%$ and $87.5 \%$ cases of bullous pemphigoid respectively. Both Banu $\mathrm{L}$ et al ${ }^{11}$ and Deepti et al ${ }^{10}$ in their study found positive DIF in all the cases of dermatitis herpetiformis.

The present study demonstrated fishnet pattern of immune complex deposits in $93.54 \%$ (29/31) cases of pemphigus vulgaris. Linear pattern of immune complex deposits were seen in all cases of bullous pemphigoid and dermatitis herpetiformis..

A positive correlation was noted between the presence of acantholytic cells in Tzanck smear and the diagnosis of pemphigus vulgaris, as these cells were identified in $100 \%(31 / 31)$ of patients within the pemphigus group of disorders. In $90.32 \%$ of cases who showed acantholytic cells in histopathology, we were able to make an early diagnosis of pemphigus vulgaris using Tzanck smear which showed acantholytic cells in $100 \%$ cases of pemphigus vulgaris.(100\% sensitivity)

A definite diagnosis of immunobullous disorders solely based on the nature of inflammatory infiltrates in cytology could not be made because of the overlapping nature of infiltrates in these disorders especially bullous pemphigoid $\&$ dermatitis herpetiformis. Further, cytological evaluation based on cellular infiltrates alone might not be confirmatory.

The sensitivity of Tzanck smear was $100 \%$ in all cases of pemphigus vulgaris as compared to DIF which was 93.54\%(29/31) Tzanck smear was negative for acantholytic cells in bullous pemhigoid and dermatitis herpetiformis, but showed predominance of eosinophils \& neutrophils respectively. The diagnosis was confirmed through histopathology and DIF in these group of disorders..

Although histopathological findings based on level of split were positive in all cases of pemphigus vulgaris and DIF was positive in $93.54 \%$ of cases, Tzanck smear was found to be more efficacious as it showed $100 \%$ sensitivity. In addition it is a simple bedside technique, which is cost effective, yields quick results and is non invasive, where as biopsy is an invasive technique, requires a skilled personal, more expensive than Tzanck smear and takes time for reporting.

\section{Conclusion}

Tzanck smear served as a simple, rapid, bedside diagnostic tool which is cost effective, can yield quick results and is a non invasive technique. It showed $100 \%$ sensitivity 
in all the cohort with pemphigus vulgaris. A definite diagnosis of immunobullous disorders solely based on the nature of inflammatory infiltrates in cytology could not be made because of the overlapping nature of infiltrates in these disorders especially in cases of bullous pemhigoid and dermatitis herpetiformis. It can be concluded that in pemphigus group of disorders, Tzanck smear is $100 \%$ sensitive and can probably serve as a first lineof investigation rather than histopathology and DIF which is expensive and takes time to yield results.

\section{Source of Funding}

None.

\section{Conflict of interest}

None.

\section{References}

1. Kirtschig G, Wojnarowska F ;

2. -. Clin Exp Dermatol. 1994;19:97-112.

3. Handa F, Agarwal RR, Garg RK. A clinical study of 85 cases of Pemphigus. Indian J Dermatol Venereol;.

4. Leena JB, Chandrashekar M, Vijaya B, Sunila R, Manjunath GV. A clinicopathological study of immunobullous lesions of the skin. A 2 years study. Adv Lab Med Int. 2012;2(2):49-60.

5. Arya SR, Valand AG, Krishna K. A clinic-pathological study of 70 cases of pemphigus. Indian J Dermatol Venereol Leprol. 1999;65:168-171.

6. Vora D, Popat V, Bhanvadia V, Mehta DA, Bhetariya B, Kumar M. A study of 75 cases of pemphigus in Saurashtra region of India. Iranian journal of dermatology. 2010;13:42-46.

7. Patel PR, Dharmesh K, Chiplonkar SG. A clinico-pathological study of 22 cases of pemphigus. National Journal Of Medical Research. 2012;2:269-271.

8. Epidemiology of pemphigus in hamedan(west of Iran), A 10 year study(1994-2004). Int J Pharm Biomed Res. 2010;1(4):157-160.
9. Leena, Vijaya B, Manjunath GV, Sunila. A clinico-pathological study of 22 cases of pemphigus. Journal of Clinical and Diagnostic Research. 2010;(4):2446-2451.

10. Deepti SP, Sulakshana MS, Manjunatha YA, Jayaprakash HT. A histomorphological study of bullous lesions of skin with special reference to immunofluorescence. Int $J$ of current research academic review;2015(3):29-51.

11. Banu L, Gulen GN, Selen S, Hulya E ; 2012,.

12. Chams-Davatchi C, Valikhani M, Daneshpazhooh M, Esmaili N, Balighi K, Hallaji Z. Pemphigus: analysis of 1209 cases. Int J Dermatol. 2005;44:470-476.

13. Kaur JS, Kanwar AJ, Kaur S, Sehgal S ; 1992,

14. Inchara YK, Rajalakshmi T. Direct immunofluorescence in cutaneous vesiculobullous lesions. Indian J Pathol Microbiol. 2007;50(4):730732.

15. Deepthi PK, Santosh CS, Suguna B, Gopal M, A. Direct immunofluorescence study in autoimmune bullous disordersof skin. IJBAR. 2013;04(09):611-618.

\section{Author biography}

\section{S Aneesh Associate Professor}

BN Raghavendra Associate Professor

Krishnan Aaryambika Resident

Anjan Kumar Patra Professor

KD Bijina Assistant Professor

Sandhaya Senior Resident

Cite this article: Aneesh S, Raghavendra BN, Aaryambika K, Patra AK, Bijina KD, S. Comparison of the diagnostic efficacy of Tzanck smear with histopathology and direct immunofluorescence in immunobullous disorders. Indian J Clin Exp Dermatol 2019;5(3):211-215. 